\title{
Editor's Note: Special Issue on Automatic Software Repair
}

(C) Springer Science+Business Media, LLC, part of Springer Nature 2018

Automatic software repair is a research field that has gained momentum over the past years. Empirical Software Engineering has contributed to fostering this movement with this special issue on Automatic Software Repair, containing the following six papers, which represent a diversity of research in this area:

- "ChangeLocator: locate crash-inducing changes based on crash reports" by Rongxin Wu, Ming Wen, Shing-Chi Cheung, and Hongyu Zhang

- "Do automated program repair techniques repair hard and important bugs?" by Manish Motwani, Sandhya Sankaranarayanan, René Just, and Yuriy Brun

- "A correlation study between automated program repair and test-suite metrics" by Jooyong Yi, Shin Hwei Tan, Sergey Mechtaev, Marcel Böhme, and Abhik Roychoudhury

- "Improved representation and genetic operators for linear genetic programming for automated program repair" by Vinicius Paulo L. Oliveira, Eduardo Faria de Souza, Claire Le Goues, and Celso G. Camilo-Junior

- "Overfitting in semantics-based automated program repair" by Xuan Bach D. Le, Ferdian Thung, David Lo, Claire Le Goues

- "Pinpointing and repairing performance bottlenecks in concurrent programs" by Tingting Yu and Michael Pradel

The journal gratefully acknowledges the authors for their contributions and the reviewers for providing valuable feedback.

The Guest Editors

Martin Monperrus

KTH Royal Institute of Technology, Sweden

Westley Weimer

University of Michigan, USA 